G. Prats · C. Roig · E. Miró • F. Navarro · B. Mirelis

\title{
In Vitro Activity of the Active Metabolite of Prulifloxacin (AF 3013) Compared with Six Other Fluoroquinolones
}

Published online: 27 April 2004

(C) Springer-Verlag 2004

\section{Eur J Clin Microbiol Infect Dis (2002) 21:328-334}

In Table 1, page 332, some of the MIC values were incorrect. The corrected values are as follows.

Microorganism Antimicrobial agent $\mathrm{MIC}(\mu \mathrm{g} / \mathrm{ml})$ MIC50 MIC90

S. pneumoniae $\mathrm{Pc}^{\mathrm{S}}(n=20) \quad \mathrm{AF} 3013$

S. pneumoniae $\mathrm{Pc}^{\mathrm{I}}(n=21) \quad \mathrm{AF} 3013$

S. pneumoniae $\mathrm{Pc}^{\mathrm{R}}(n=17)$ AF 3013

14

E. faecium $\mathrm{Vm}^{\mathrm{R}}(n=7)$ moxifloxacin

24

22

Pc, penicillin; Vm, vancomycin; ${ }^{\mathrm{S}}$, susceptible; ${ }^{\mathrm{R}}$, resistant; ${ }^{\mathrm{I}}$, intermediate doi.org/10.1007/s10096-002-0709-1

G. Prats $(\bowtie) \cdot$ C. Roig · E. Miró · F. Navarro · B. Mirelis Servei de Microbiologia, Hospital de la Santa Creu i Sant Pau, Avenida Sant Antoni Ma Claret, 167, 08025 Barcelona, Spain,

e-mail: gprats@cs.vhebron.es

Tel.: +34-93-2919071

Fax: +34-93-2919070 